\title{
Study of Thermoelastic behaviour, Efficiency and Effectiveness of Rectangular Fin with Fractional Order Energy Balance Equation
}

\author{
J.J. Bikram ${ }^{1, *}$, G.D. Kedar ${ }^{2}$ \\ ${ }^{1,2}$ Department of Mathematics, \\ RTM Nagpur University, \\ Nagpur-440 033 (Maharashtra) India. \\ E-mail: 1jayabikram@gmail.com
}

Received 13 February 2021, Revised 31 March 2021, Accepted 5 May 2021

\begin{abstract}
In this paper, we have considered the Caputo fractional order model for convective fins of rectangular profile with temperature-dependent thermal conductivity. The fractional order energy balance equation is solved by using homotopy perturbation method (HPM). This method is one of the effective tools to solve the fractional order nonlinear diffusion equation with thermosensitive conductivity it requires less computer memory and reduces the computation time. The fin efficiency and the fin effectiveness appeared as a function of thermo-geometric fin parameters. The stresses are solved using stress-displacement relation. The results obtained are illustrated graphically for temperature distribution, efficiency, effectiveness and thermal stresses. The phenomena reveal that the selection of the order of fractional derivative remarkably influences the outcomes. However, careful review of the existing literature reveals that hardly few results of thermal stresses in fins with the energy balance equation of integer order are available. Hence this result may be the novel contribution to the field.
\end{abstract}

Keywords: Fractional order thermoelasticity; Homotopy Perturbation Method; Fin efficiency; Rectangular fin.

\section{Introduction}

The heat transfer is a very attractive field in various branches of engineering and has huge applications in mechanical engineering. The process of heat transfer can be improved by using the extended surface which is called fin. The rate of heat dissipation can be increased exposing the fin to cool or heat with the high thermal conductivity. Various methods had been empowered to solve the heat transfer problem of the fins. Aziz and Huq [1] used perturbation method to calculate the closed form solution for a straight convective fin with thermosensitive thermal conductivity. Besides that, the Fourier series method approach used to investigate a two-dimensional rectangular fin with arbitrary variable heat transfer coefficient on the fin surface [2]. The obtained solutions show that the temperature distribution with three different boundary conditions at the fin tip. Further investigation leads towards parametric study of horizontal fin arrays, the threedimensional elliptic governing equations solved using a finite computational fluid dynamics code [3]. The obtained results showed that it is not possible to obtain optimum performance in terms of heat transfer by only concentrating on one or two parameters. Mokheimer [4] investigated the performance of annular fins of different profiles subject to locally variable heat transfer coefficient. The divergence between the fin efficiency calculated based on constant and variable heat transfer coefficient, has been estimated and presented for all fin profiles with different radius ratios. More theory came forward which showed the effect of the fin arrays geometries and also the fin tip-to-shroud clearance on the heat transfer, the fluid flow and the pressure drop characteristics of longitudinal rectangular-fin arrays [5]. Chiu and Chen [6] applied Adomian's decomposition method and discussed fin efficiency. Arslanturk [7] analysed fin efficiency of convective straight fins with the help of decomposition method. The analysis to the fin theory extended to calculate the rate of entropy generation of pin fins of circular and elliptical cross flow of air. Coskun and Atay [8] operated the variational iteration technique to examine the convective straight and radial fins. The study shows that the competition between enhanced thermal contact and fluid friction is settled when the heat transfer irreversibility and the fluid friction irreversibility add [9]. Domairry and Fazeli [10] also determined the fin efficiency with the help of Homotopy analysis method. Cuce and Cuce [11] applied Homotopy perturbation method to discuss fin efficiency and its effectiveness in straight fin. The profile of the extended surface varies as the thermal behaviour of the system also changes. Moreover, [12] illustrated the thermodynamics optimization of a Y shaped fin profile to study the latent heat thermal storage system. The result represents the detailed thermodynamics optimization of a system involving an unsteady process. Furthermore, Huang et. al [13] obtained the solution to improve ventilation with cold air from below the fin base by introducing the perforations through the fin base. Mallick et. al [14] determined analytically the thermal stresses in an annular convective-conductive fin of hyperbolic profile with steady state energy balance equation in the form of integer order derivatives. 
The generalization of the derivatives and integrations of non-integer orders are called Fractional derivatives and integrations [15]. Due to the non-local property of fractional derivatives the researchers are using it in various fields of physical sciences such as Electromagnetism, quantum mechanics and fluid dynamics. In the field of thermoelasticity, the heat diffusion process in heterogeneous and non-regular materials the transport processes do not obey the law of classical mechanics. Hence, it becomes necessary to consider the diffusion equation with fractional order derivatives. Although Fractional order theory has been studied in great detail, Caputo derivative $[16,17,18]$ achieves more importance as it easily assembles to heat conduction equations of nonlinear order. This new method is adopted to solve the thermoelastic problem related to the field of mechanics $[19,20,21,22]$. Srinivas [23] considered the finite rectangular parallelepiped subjected to conducting heating with time fractional order heat conduction equation of Caputo derivative form. The effect of time fractional parameters on temperature distribution, deflection, stress resultants and thermal stress distribution has been observed. Further theory has been extended to study the theory of fin with fractional order. Patel and Meher [24] studied the variation of temperature distribution, efficiency and effectiveness of porous fin for different fractional order, porous parameter and convection parameter by using Adomian Decomposition Sumudu Transform Method(ADSTM). Patra and Saha Ray [25] used Homotopy Perturbation Method to analyse fin efficiency using fractional order energy balance equation. These researchers analysed the fin efficiency of convective straight fin. Devendra [26] employed the HPM coupled with Laplace transform method to obtained the solution of fractional order problem for convective straight fins with temperature-dependent thermal conductivity associated with Caputo-Fabrizio fractional derivative.

In this paper, we have considered the Fourier law in the form of fractional order derivatives to study the efficiency and effectiveness of a rectangular fin. The temperature distribution is determined using the HPM and thermal stresses are obtained simplifying stress-displacement relation. The results obtained are illustrated graphically for better understanding the effect of thermal geometric fin parameter, thermal conductivity on temperature distribution, thermal stresses for different fractional parameters and also fin efficiency and effectiveness with respect to thermal geometric fin parameter. However, careful review of the existing literature reveals that hardly few results of thermal stresses in fins are available with the energy balance equation of integer order. Hence this result may be the novel contribution to the field.

\section{Problem Formulation of Heat Transfer}

We have considered a rectangular fin with constant cross-sectional area $A_{c}$, perimeter $P$, length $b$ and heat flows in $x$ direction. The fin is subjected to base surface temperature $T$ and extended into the fluid at ambient temperature $T_{a}$ (see in Figure 1). The tip of the fin is assumed to be insulated. The heat flow enters the fin is $Q_{\text {in }}=Q$ and flow out of fin is $Q_{\text {out }}=Q+\frac{d Q}{d x} d x$, while some heat flows due to convection is
$\operatorname{Pdxh}\left(T-T_{a}\right)$, where $h$ is heat transfer coefficient from surface to surrounding and $x$ is the spatial variable. The steady state energy balance performed on this elementary area is given by $[4,27]$

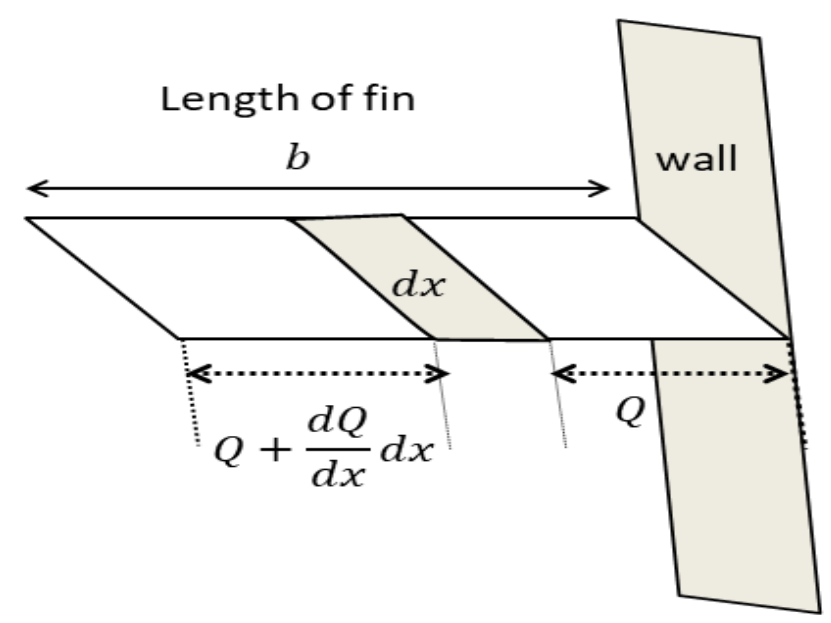

Figure 1. Representation of the rectangular fin.

$Q_{\text {in }}=Q_{\text {out }}+\operatorname{pdxh}\left(T-T_{a}\right)$

Introducing $Q=q A_{c}$ in equation (1) reduces to

$A_{c} \frac{d q}{d x}+p h\left(T-T_{a}\right)=0$

Using the non-local generalizations of the Fourier Law as in [28]

$q=-K(T) \frac{d^{\alpha} T}{d x^{\alpha}}$

The thermal conductivity $K(T)$ of the fin is taken as temperature dependent

$K(T)=k_{a}\left[1+\lambda\left(T-T_{a}\right)\right]$

where $\lambda$ is a parameter defining the variation of the thermal conductivity, $k_{a}$ is the thermal conductivity at the ambient fluid temperature of the fin.

Substitute the equation (3) in (2), its leads to fractional order energy balance equation for rectangular fin

$$
\begin{array}{r}
\frac{d}{d x}\left[A_{c} K(T) \frac{d^{\alpha} T}{d x^{\alpha}}\right]-p h\left(T-T_{a}\right)=0 \\
0 \leq x \leq b \quad 0<\alpha \leq 1
\end{array}
$$

For simplicity the non-dimensional parameters are defined as

$$
\begin{aligned}
& \xi=\frac{x}{b}, \quad \beta=\lambda\left(T_{b}-T_{a}\right), \quad \theta=\frac{T-T_{a}}{T_{b}-T_{a}}, \\
& \phi^{2}=\left(\frac{h P b^{\alpha}}{k_{a} A_{c}}\right)
\end{aligned}
$$

where $\beta$ is a non-dimensional thermal conductivity parameter, $\phi$ is thermo-geometric fin parameter and $T_{b}$ is the fin base temperature.

By introducing dimensionless quantities (6) in energy balance equation (5) one obtains, 


$$
\begin{gathered}
\frac{d^{\alpha+1} \theta}{d \xi^{\alpha+1}}+\beta \theta \frac{d^{\alpha+1} \theta}{d \xi^{\alpha+1}}+\beta\left(\frac{d \theta}{d \xi} \frac{d^{\alpha} \theta}{d \xi^{\alpha}}\right)-\phi^{2} \theta=0 \\
0 \leq \xi \leq 1
\end{gathered}
$$

In order to determine temperature distribution the following boundary conditions are considered

$$
\begin{aligned}
& \xi=0 ; \quad \frac{d \theta}{d \xi}=0 \\
& \xi=1 ; \quad \theta=1
\end{aligned}
$$

\section{Essentials of Fractional Calculus}

Fractional calculus has achieved the tremendous rise in the field of physics and engineering as it supports the fractional order differential and integral equation. The vast literatures are available for definitions of fractional derivatives. In this work, we are using Caputo fractional derivative as it allows traditional initial and boundary conditions in the formation of the problem and it also shows the derivative of constant is zero.

The Caputo definition of fractional derivatives of $\theta(\xi)$ of order $\alpha$ is defined by [29]

$$
\begin{aligned}
& D_{C}^{\alpha} \theta(\xi)=I^{n-\alpha} D^{n} \theta(\xi) \\
& =\frac{1}{\Gamma(n-\alpha)} \int_{0}^{\xi}(\xi-t)^{n-\alpha-1} \frac{d^{n} \theta(\xi)}{d t^{\alpha}} d t \\
& \xi>0 \quad n-1<\alpha<n
\end{aligned}
$$

where $D=\frac{d}{d \xi}$ is the derivative operator, $n$ is a positive integer. If $\theta(\xi)=\xi^{\gamma}$ then Caputo definition leads to

$$
D^{\alpha} \xi^{\gamma}=\frac{\Gamma(\gamma+1)}{\Gamma(\gamma-\alpha+1)} \xi^{\gamma-\alpha} \quad \gamma>-1
$$

Linearity property of integer order differential equation is

$$
D^{\alpha}(z f(\theta)+r g(\theta))=z D^{\alpha} f(\theta)+r D^{\alpha} g(\theta)
$$

Where $f(\theta), g(\theta)$ are derivative terms of $\theta$ and $z, r$ are constant integers. The fractional integrals of a function $\xi^{\gamma}$ is

$$
D^{-\alpha} \xi^{\gamma}=\frac{\Gamma(\gamma+1)}{\Gamma(\gamma+\alpha+1)} \xi^{\gamma+\alpha} \quad \gamma>-1 \quad \alpha>0
$$

In particular, if $\gamma=0$, the fractional integral of a constant

$C$ of order $\alpha$ is

$$
D^{-\alpha} C=\frac{C}{\Gamma(\alpha+1)} \xi^{\alpha} \quad \alpha>0
$$

\section{Homotopy Perturbation Method To Heat Transfer} HPM is used to obtain approximate analytic solution of nonlinear differential equation as in [30],[31],[32]. We have considered a non-linear differential equation of the form

$$
A(\theta)-f(\xi)=0
$$

where $f(\xi)$ an analytic known function and $A$ is separated into two parts as

$$
A(\theta)=L(\theta)+N(\theta)
$$

where $L(\theta)$ is a linear part and $N(\theta)$ is non-linear part of the differential equation. By homotopy technique one can construct a homotopy $H(\theta, s)$ which satisfies:

$$
H(\theta, s)=(1-s)\left[L(\theta)-L\left(\theta_{0}\right)\right]+s[A(\theta)-f(\xi)]=0
$$

From this equation (17) we can have the condition, where $s \in[0,1]$ is an embedding parameter and $\theta_{0}$ is the first approximation of equation (15) that satisfies the boundary conditions. Hence, we have

$$
\begin{aligned}
& H(\theta, 0)=L(\theta)-L\left(\theta_{0}\right)=0 \\
& H(\theta, 1)=A(\theta)-f(\xi)=0
\end{aligned}
$$

As $s$ changes from zero to unity the quantity of $\theta(\xi, s)$ changes from $\theta_{0}(\xi)$ to $\theta(\xi)$. This process is known as deformation and $L(\theta)-L\left(\theta_{0}\right) \quad$ and $L(\theta)+N(\theta)-f(\xi)$ are the homotopy. Applying classical perturbation technique we assume the solution $\theta$ of equation (17) as a power series

$\theta=\sum_{i=0}^{l} s^{i} \theta_{i}=\theta_{0}+s \theta_{1}+s^{2} \theta_{2}+s^{3} \theta_{3}+\cdots$

In this method the values of $\theta_{0}, \theta_{1}, \theta_{2}, \theta_{3} \cdots$ are evaluated by the function obtained from (17),(18) and (19). Finally, the approximation for solution can be obtained by

$\theta=\lim _{s \rightarrow 1} \sum_{i=0}^{l} s^{i} \theta_{i}$

This series converges very rapidly. Applying homotopy perturbation method, the linear and non-linear parts of (7) are termed as

$$
\begin{aligned}
& L(\theta)=\frac{d^{\alpha+1} \theta}{d \xi^{\alpha+1}} \\
& N(\theta)=\beta \theta \frac{d^{\alpha+1} \theta}{d \xi^{\alpha+1}}+\beta\left(\frac{d \theta}{d \xi} \frac{d^{\alpha} \theta}{d \xi^{\alpha}}\right)-\phi^{2} \theta
\end{aligned}
$$

The insulated boundary condition (8) gives us $\theta$ as an arbitrary constant at $\xi=0$. Hence this boundary condition can be rewrite as

$\frac{d \theta}{d \xi}=0$ at $\xi=0 ; \quad \theta=C$ at $\xi=0$

Inserting (20) into (22) and then in (17) and then from obtained results equating the coefficients of power of $S$, we get

$$
s^{0}: \frac{d^{\alpha+1} \theta_{0}}{d \xi^{\alpha+1}}=0
$$


with $\frac{d \theta_{0}}{d \xi}=0$ at $\xi=0 ; \quad \theta_{0}=C$ at $\xi=0$

$$
=C+C \frac{\xi^{\alpha}}{\Gamma(\alpha+1)}+\left(\varphi^{2} C-\beta \varphi^{2} C^{2}+\beta^{2} C^{3} \varphi^{2}\right) \frac{\xi^{\alpha+1}}{\Gamma(\alpha+2)}
$$

$s^{1}: \frac{d^{\alpha+1} \theta_{1}}{d \xi^{\alpha+1}}+\beta \theta_{0} \frac{d^{\alpha+1} \theta_{0}}{d \xi^{\alpha+1}}+\beta\left(\frac{d \theta_{0}}{d \xi} \frac{d^{\alpha} \theta_{0}}{d \xi^{\alpha}}\right)-\phi^{2} \theta_{0}=0+\left(\varphi^{2} C-\beta \varphi^{2} C^{2}-\varphi^{2} C^{2}+\beta C^{3} \varphi^{2}+2 \beta^{2} C^{3} \varphi^{2}\right) \frac{\xi^{2 \alpha+1}}{\Gamma(2 \alpha+2)}$

with $\frac{d \theta_{1}}{d \xi}=0$ at $\xi=0 ; \quad \theta_{1}=0 \quad$ at $\xi=0$

$$
\begin{aligned}
& s^{2}: \frac{d^{\alpha+1} \theta_{2}}{d \xi^{\alpha+1}}+\beta \theta_{0} \frac{d^{\alpha+1} \theta_{1}}{d \xi^{\alpha+1}}+\beta \theta_{1} \frac{d^{\alpha+1} \theta_{0}}{d \xi^{\alpha+1}} \\
& +\beta\left(\frac{d \theta_{0}}{d \xi} \frac{d^{\alpha} \theta_{1}}{d \xi^{\alpha}}\right)+\beta\left(\frac{d \theta_{1}}{d \xi} \frac{d^{\alpha} \theta_{0}}{d \xi^{\alpha}}\right)-\phi^{2} \theta_{1}=0
\end{aligned}
$$

with $\frac{d \theta_{2}}{d \xi}=0$ at $\xi=0 ; \quad \theta_{2}=0$ at $\xi=0$

$$
\begin{aligned}
s^{3}: \frac{d^{\alpha+1} \theta_{3}}{d \xi^{\alpha+1}} & +\beta \theta_{0} \frac{d^{\alpha+1} \theta_{2}}{d \xi^{\alpha+1}}+\beta \theta_{1} \frac{d^{\alpha+1} \theta_{1}}{d \xi^{\alpha+1}}+\beta \theta_{2} \frac{d^{\alpha+1} \theta_{0}}{d \xi^{\alpha+1}} \\
& +\beta\left(\frac{d \theta_{0}}{d \xi} \frac{d^{\alpha} \theta_{2}}{d \xi^{\alpha}}\right)+\beta\left(\frac{d \theta_{1}}{d \xi} \frac{d^{\alpha} \theta_{1}}{d \xi^{\alpha}}\right) \\
& +\beta\left(\frac{d \theta_{2}}{d \xi} \frac{d^{\alpha} \theta_{0}}{d \xi^{\alpha}}\right)-\phi^{2} \theta_{2}=0
\end{aligned}
$$

$$
+\left(2 \beta \varphi^{2} C^{3}+\beta^{2} C^{3} \varphi^{2}-C^{3} \varphi^{2}\right) \frac{\Gamma(2 \alpha+1) \xi^{3 \alpha+1}}{\Gamma(\alpha+1) \Gamma(\alpha+1) \Gamma(3 \alpha+2)}
$$$$
+\left(\frac{\beta C^{3}}{\Gamma(\alpha+1) \Gamma(\alpha+1)}-\frac{2 \beta^{2} C^{4}}{\Gamma(\alpha+1) \Gamma(\alpha+1)}\right) \frac{\Gamma(2 \alpha+2) \xi^{3 \alpha+2}}{\Gamma(3 \alpha+3)}
$$$$
+\left(\frac{\beta C^{3}}{\Gamma(\alpha+1)}-\frac{\beta C^{2}}{\Gamma(\alpha+1)}-\frac{\beta^{2} C^{4}}{\Gamma(\alpha+1)}-\frac{\beta C^{2} \phi^{4}}{\Gamma(\alpha+2)}\right) \frac{\Gamma(\alpha+2) \xi^{2 \alpha+2}}{\Gamma(2 \alpha+3)}
$$$$
-\left(\frac{C \phi^{4}}{\Gamma(2 \alpha+2)}+\frac{\beta C^{2} \phi^{4}}{\Gamma(2 \alpha+2)}\right) \frac{\Gamma(2 \alpha+2) \xi^{3 \alpha+2}}{\Gamma(3 \alpha+3)}+\cdots
$$

where $C$ represents the temperature at the fin tip which can be determined by using boundary condition (9).

\section{Fin Efficiency and Effectiveness}

with $\frac{d \theta_{3}}{d \xi}=0$ at $\xi=0 ; \quad \theta_{3}=0 \quad$ at $\xi=0$

$s^{4}: \frac{d^{\alpha+1} \theta_{4}}{d \xi^{\alpha+1}}+\beta \theta_{0} \frac{d^{\alpha+1} \theta_{3}}{d \xi^{\alpha+1}}+\beta \theta_{1} \frac{d^{\alpha+1} \theta_{2}}{d \xi^{\alpha+1}}+\beta \theta_{2} \frac{d^{\alpha+1} \theta_{1}}{d \xi^{\alpha+1}} \quad \eta=\frac{q_{a}}{q_{i}}$

$+\beta \theta_{3} \frac{d^{\alpha+1} \theta_{0}}{d \xi^{\alpha+1}}+\beta\left(\frac{d \theta_{0}}{d \xi} \frac{d^{\alpha} \theta_{3}}{d \xi^{\alpha}}\right)+\beta\left(\frac{d \theta_{1}}{d \xi} \frac{d^{\alpha} \theta_{2}}{d \xi^{\alpha}}\right)$

where the heat dissipation from the fin surface is evaluated by Newton's law of cooling as

$+\beta\left(\frac{d \theta_{2}}{d \xi} \frac{d^{\alpha} \theta_{1}}{d \xi^{\alpha}}\right)+\beta\left(\frac{d \theta_{3}}{d \xi} \frac{d^{\alpha} \theta_{0}}{d \xi^{\alpha}}\right)-\phi^{2} \theta_{3}=0$

$q_{a}=\int_{0}^{b} P\left(T-T_{a}\right) d x$

with $\frac{d \theta_{4}}{d \xi}=0$ at $\xi=0 ; \quad \theta_{4}=0 \quad$ at $\xi=0$

Thus, proceeding in the same manner by equating coefficients of powers of $S$ we get differential equations for remaining components $\theta_{2}, \theta_{3}, \theta_{4} \cdots$. The desired accuracy can be obtained by increasing the number of terms sufficiently in the series solution.

Solving the differential equation (24)-(28) for $\theta_{0}, \theta_{1}, \theta_{2}, \theta_{3}, \theta_{4} \cdots$ and letting $s \rightarrow 1$, we have obtained the solution $\theta(\xi)$ as

and ideal heat transfer rate is given by

$q_{i}=P b\left(T_{b}-T_{a}\right)$

Taking eqs. (31) and (32) in considerations, the fin efficiency given in equation (30) takes the dimensionless form according to (6) as

$\eta=\int_{0}^{1} \theta(\xi) d \xi$

The effectiveness of an fin can be determined by [27]

$\omega=\frac{q_{a}}{q_{b}}=\frac{\int_{0}^{b} 2 h P\left(T-T_{a}\right) d x}{\operatorname{Pht}\left(T_{b}-T_{a}\right)}$ 
In dimensionless form fin effectiveness becomes

$\omega=\int_{0}^{1} \psi \phi(\xi) d \xi$

where $q_{b}$ is the rate of heat loss from the fin base, $t$ is the thickness of the fin which is assume to be smaller than the width $W$ of the fin and $\psi=\frac{2 b}{t}$.

\section{Thermal Stress}

The stress-displacement relations for axisymmetric case [33],[34] are

$$
\begin{aligned}
& \sigma_{y y}(x, t)=\frac{(1-v) E}{(1+v)(1-2 v)}\left[\frac{v}{1-v} \frac{d u}{d x}-\frac{1+v}{1-v} a_{T} T\right] \\
& \sigma_{x x}(x, t)=\frac{(1-v) E}{(1+v)(1-2 v)}\left[\frac{d u}{d x}-\frac{1+v}{1-v} a_{T} T\right]
\end{aligned}
$$

where $\sigma_{x x}, \sigma_{y y}$ are stress components, $u$ is th displacement component in $x$ direction, $a_{T}$ is th coefficient of linear thermal expansion, $E$ denotes th Young's modulus and $\eta$ is the Poisson's ratio. Th equilibrium equation in absence of body force along $\lambda$ direction is

$$
\frac{d \sigma_{x x}}{d x}=0
$$

The eqs. (36) and (37) yield the equation of equilibrium i terms of the displacement field

$$
\frac{d^{2} u}{d x^{2}}=\frac{1+v}{1-v} a_{T} \frac{d T}{d x}
$$

with boundary conditions:

at $x=0, \quad u=0$,

at $x=1, \quad \frac{d u}{d x}=\frac{1+v}{1-v} a_{T} \frac{d T}{d x}$

Introducing a non-dimensional parameters $\bar{u}=\frac{(1-v) u}{b a_{T}(1+v)}$ the equilibrium equation reduced to

$\frac{d^{2} \bar{u}}{d \xi^{2}}=\frac{\beta}{\lambda} \frac{d \theta}{d \xi}$

And $\left(\bar{\sigma}_{x x}, \bar{\sigma}_{y y}\right)=\frac{(1-2 v)}{E a_{T}}\left(\sigma_{x x}, \sigma_{y y}\right)$ are the nondimensional components to modify the stress-displacement relation in new form

$$
\bar{\sigma}_{x x}=\frac{d \bar{u}}{d \xi}-\left[\frac{\beta}{\lambda} \theta+T_{a}\right]
$$

$$
\bar{\sigma}_{y y}=\frac{v}{1-v} \frac{d \bar{u}}{d \xi}-\left[\frac{\beta}{\lambda} \theta+T_{a}\right]
$$

where non-dimensional boundary conditions are

at $\xi=0, \frac{d}{d \xi}\left(\bar{\sigma}_{x x} ; \bar{\sigma}_{y y}\right)=0$

at $\xi=1,\left(\bar{\sigma}_{x x} ; \bar{\sigma}_{y y}\right)=\frac{(2 v-1)}{E a_{T}} \frac{\beta}{\lambda}-T_{a}$

The approximate solution for the stress field is employed from (41), where the constant is estimated from (42).

Table 1: The comparative study of non-dimensional temperature distribution of fin for different values of fractional order $\alpha$ at $\beta=1$ and $\phi=1$.

\begin{tabular}{|c|c|c|c|c|}
\hline \multirow{2}{*}{$\xi$} & \multicolumn{4}{|c|}{ HPM } \\
\cline { 2 - 5 } & $\alpha=0.25$ & $\alpha=0.5$ & $\alpha=0.75$ & $\alpha=1$ \\
\hline 0 & 0.2649 & 0.327 & 0.4014 & 0.4851 \\
\hline 0.1 & 0.289409058 & 0.340827717 & 0.408981265 & 0.489080704 \\
\hline 0.2 & 0.326498411 & 0.367408253 & 0.427360891 & 0.501170905 \\
\hline 0.3 & 0.37337867 & 0.404293619 & 0.455545154 & 0.521810647 \\
\hline 0.4 & 0.430009935 & 0.451537127 & 0.493858337 & 0.551723971 \\
\hline 0.5 & 0.496751785 & 0.509765449 & 0.543123313 & 0.591910167 \\
\hline 0.6 & 0.574088462 & 0.579853673 & 0.604469603 & 0.643634337 \\
\hline 0.7 & 0.662549623 & 0.662809159 & 0.679247569 & 0.708417295 \\
\hline 0.8 & 0.762680759 & 0.759718078 & 0.768979502 & 0.788024783 \\
\hline 0.9 & 0.875030683 & 0.871716977 & 0.875327502 & 0.884456007 \\
\hline 1 & 1.000145946 & 0.99997617 & 1.000070283 & 0.999931502 \\
\hline
\end{tabular}

order of $\alpha$. This method provides a solution as a polynomial expression and the main advantage of the method is that it reduces the computation time. Table 1. show the non-dimensional distribution of temperature with $\xi$ for different values of fractional order $\alpha$. It is observed that the temperature of the fin increases with rise in fractional order. Thus the cooling or heating of fin can be managed by selecting $\alpha$.

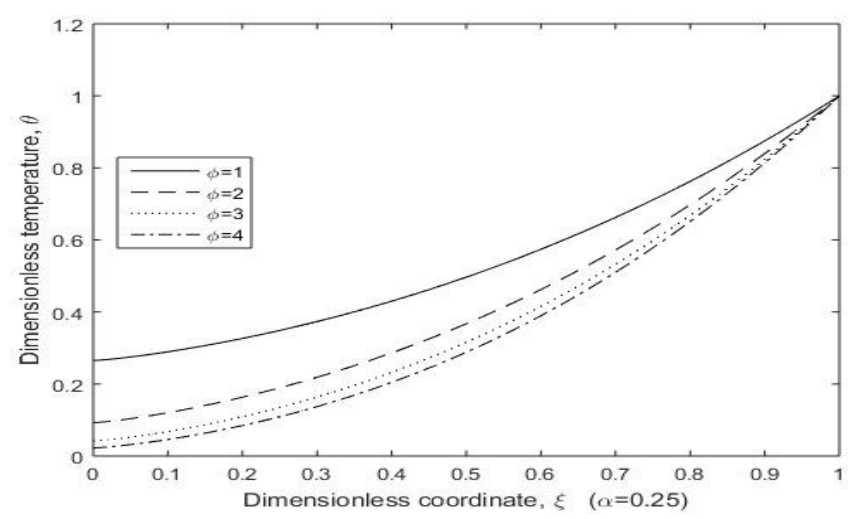

(a) $\alpha=0.25$ 


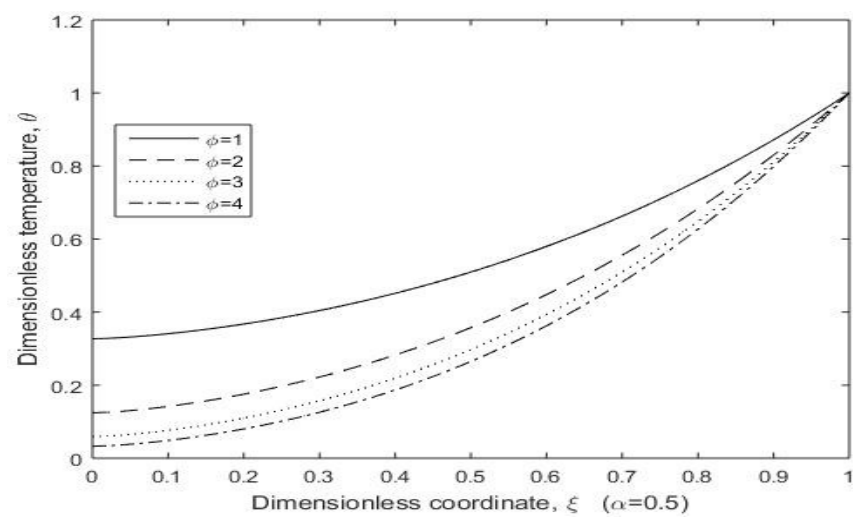

(b) $\alpha=0.5$

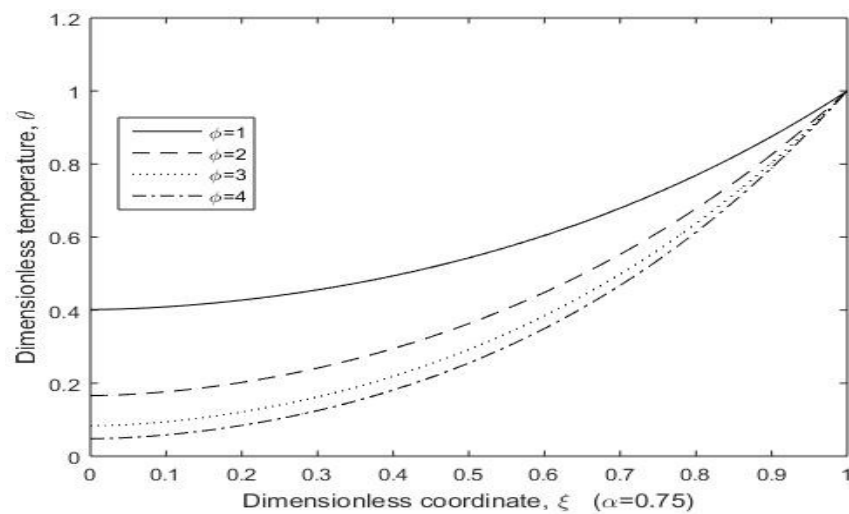

(c) $\alpha=0.75$

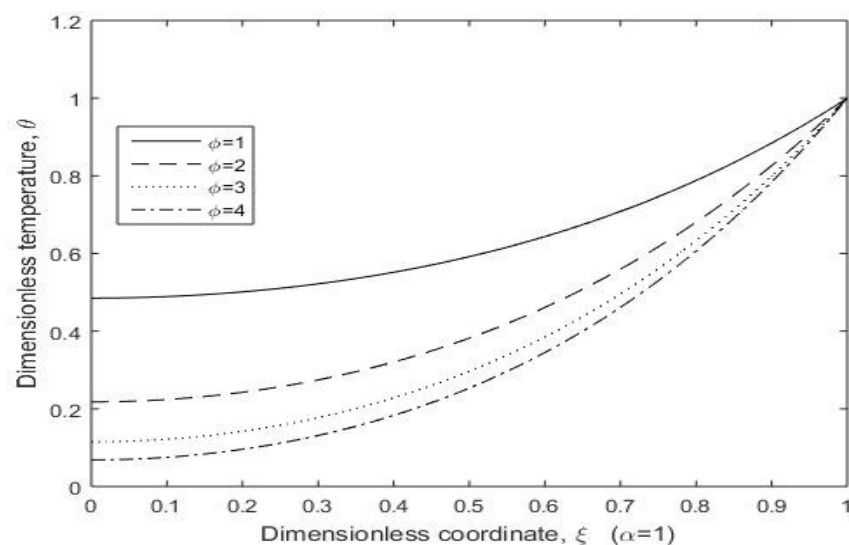

(d) $\alpha=1$

Figure 2: Dimensionless Temperature distribution with space variable $\xi$ for different values of thermal conductivity parameter $\beta$ and fractional order $\alpha$.

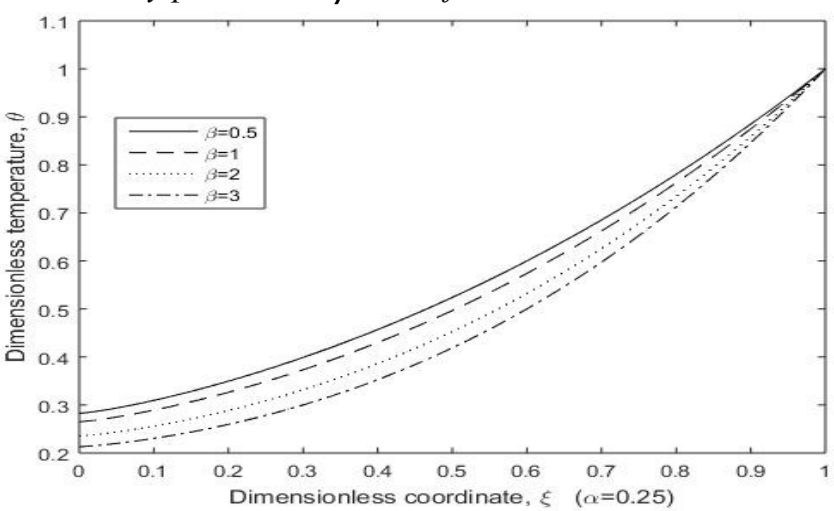

(a) $\alpha=0.25$

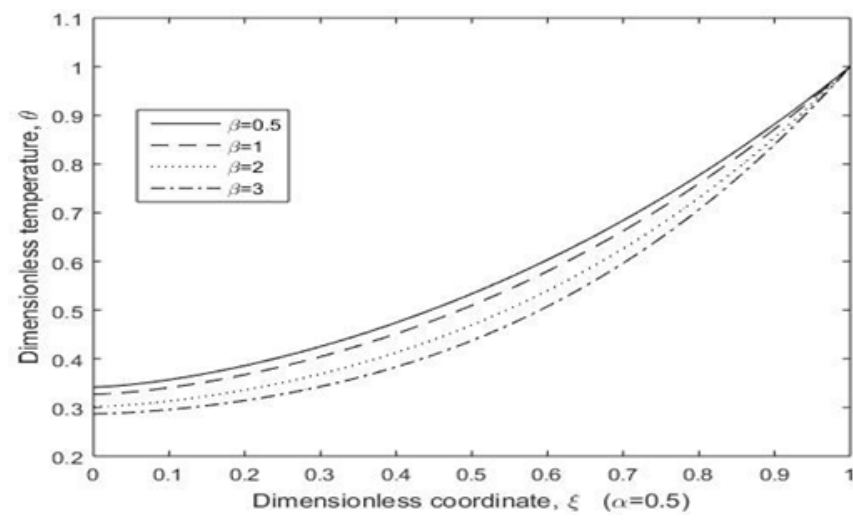

(b) $\alpha=0.5$

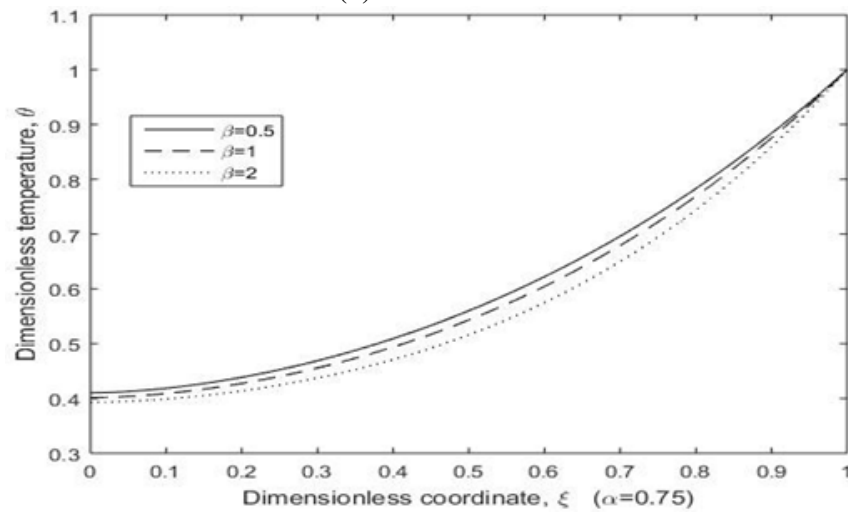

(c) $\alpha=0.75$

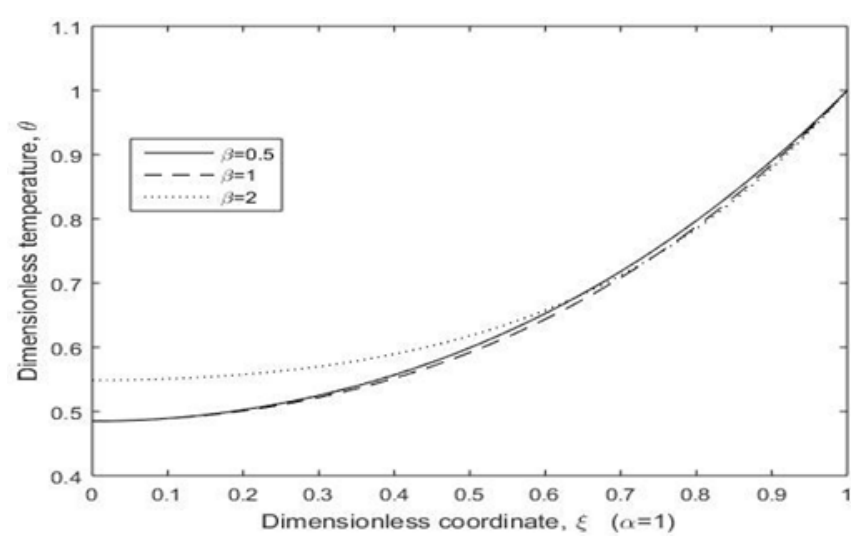

(d) $\alpha=1$

Figure 3: Dimensionless Temperature distribution with space variable $\xi$ for different values of thermal conductivity parameter $\beta$ and fractional order $\alpha$.

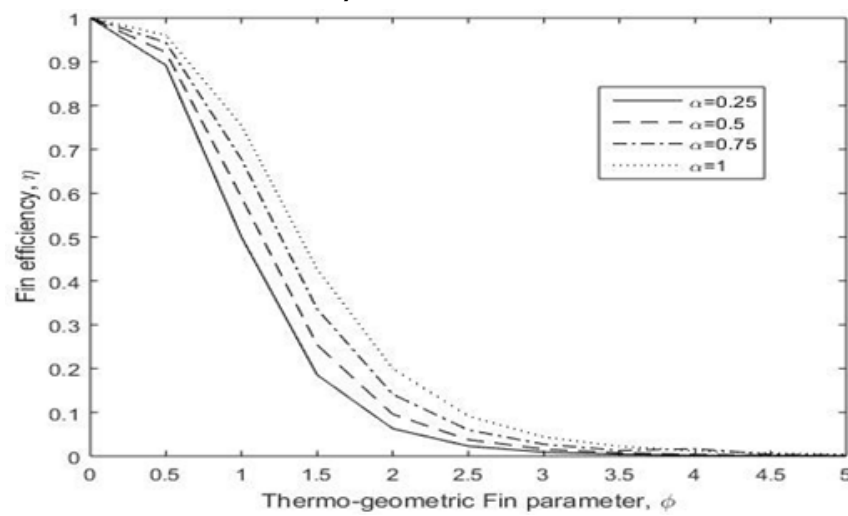

Figure 4: Dimensionless fin efficiency for different values of thermo-geometric fin parameters $\phi$ and fractional order $\alpha$. 


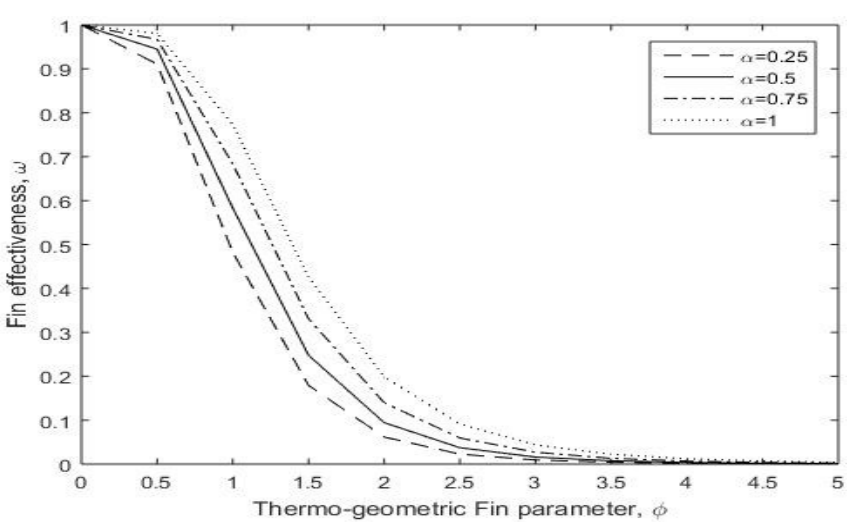

(a) $\beta=1$

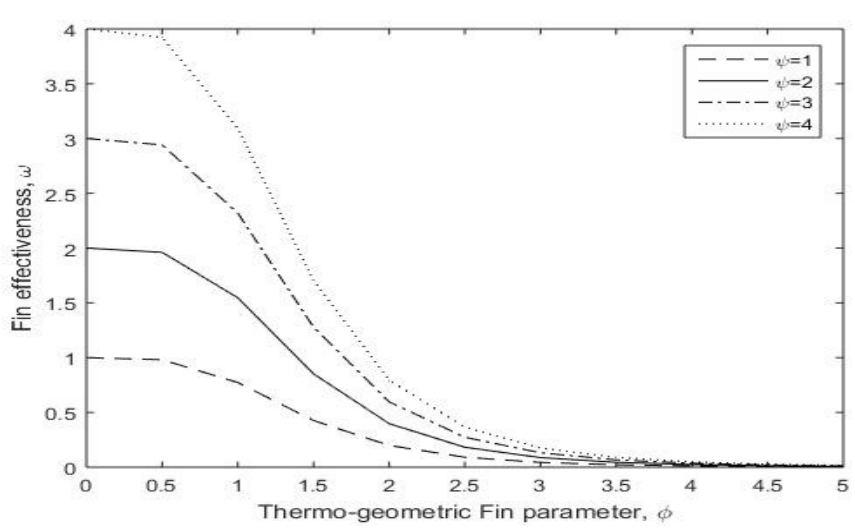

(b) $\beta=1, \alpha=1$

Figure 5: Dimensionless fin effectiveness for different values of (a) fractional order $\alpha$ and (b) fin length/fin thickness ratio $\psi$.

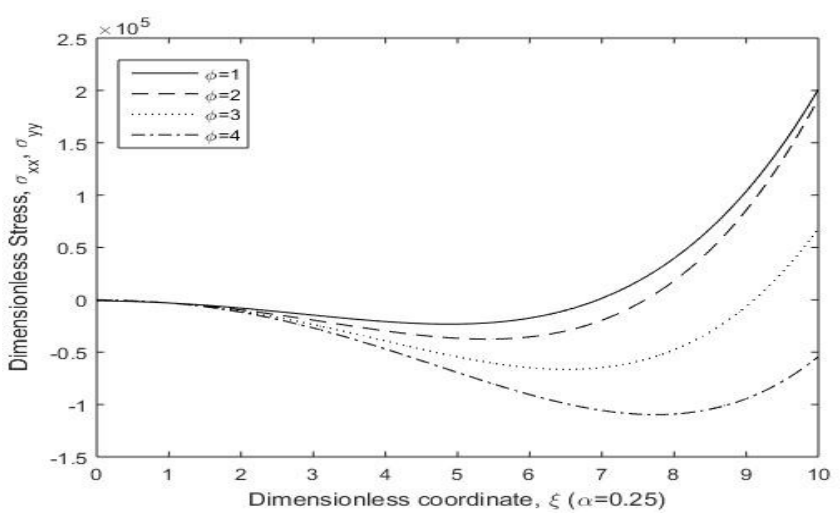

(a) $\alpha=0.25$

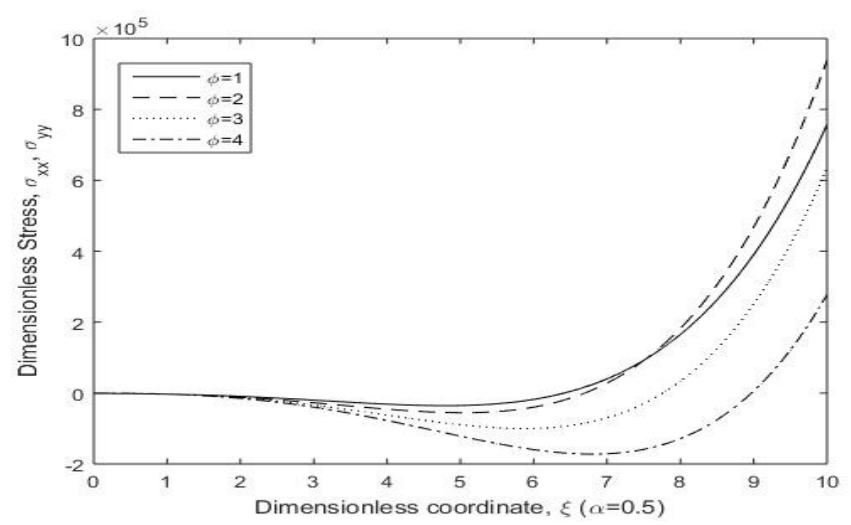

(b) $\alpha=0.5$

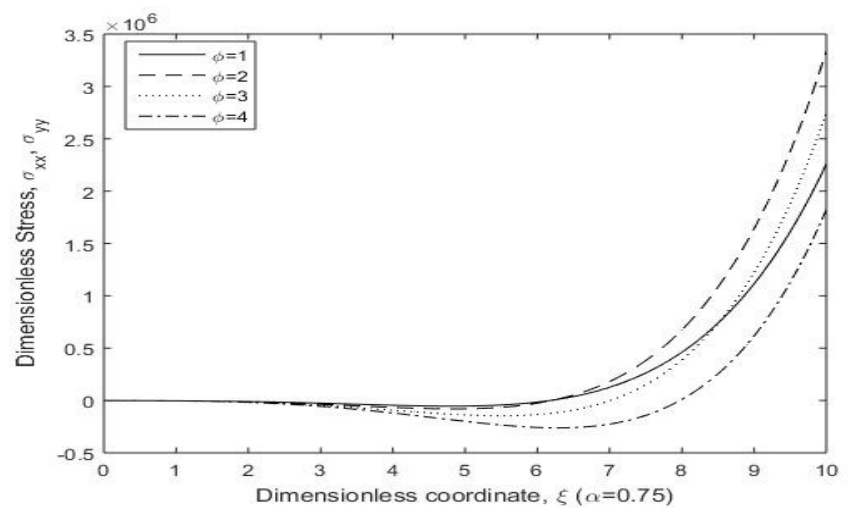

(c) $\alpha=0.75$

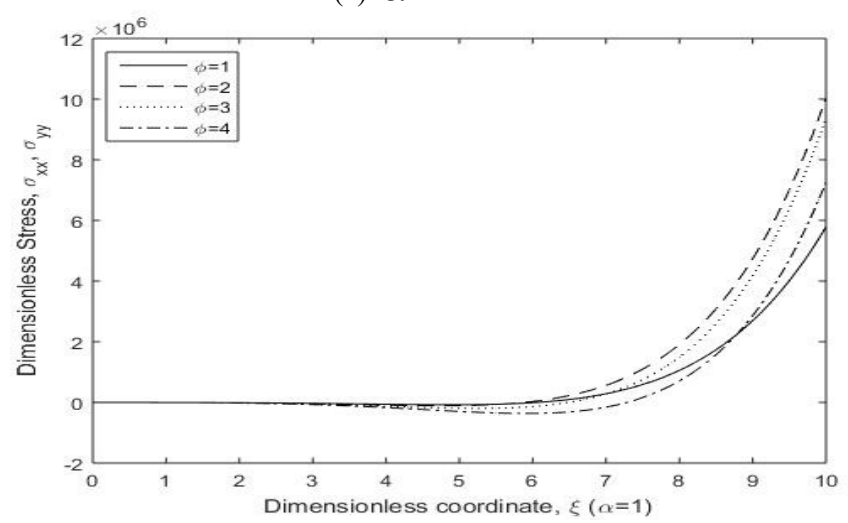

(d) $\alpha=1$

Figure 6: Dimensionless Stress distribution with space variable $\xi$ for different values of thermo-geometric fin parameters $\phi$ and fractional order $\alpha$.

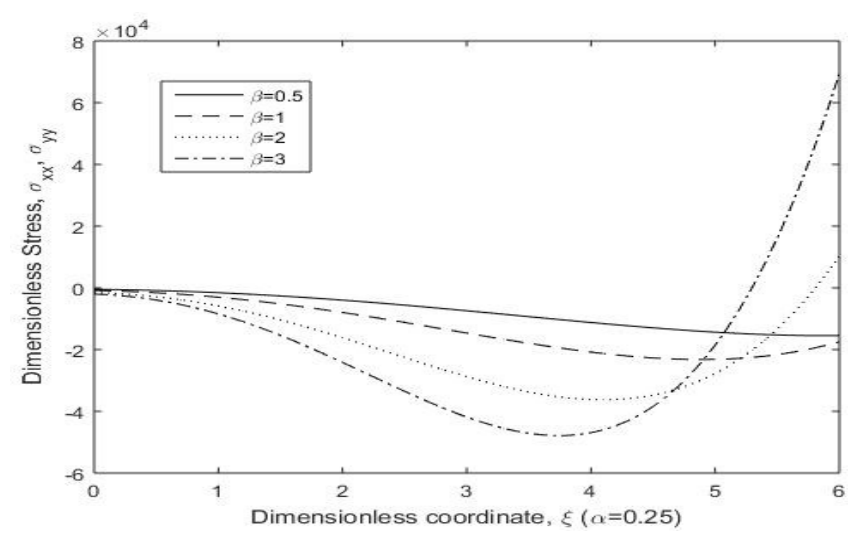

(a) $\alpha=0.25$ 


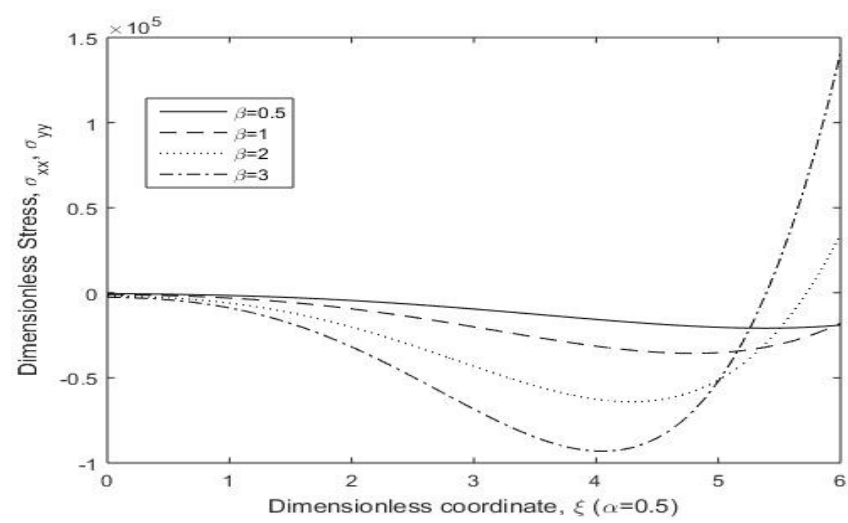

(b) $\alpha=0.5$

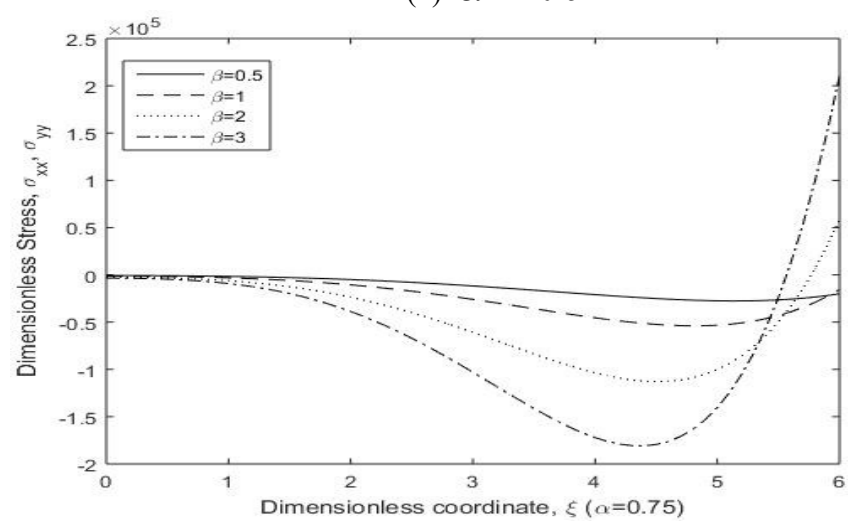

(c) $\alpha=0.75$

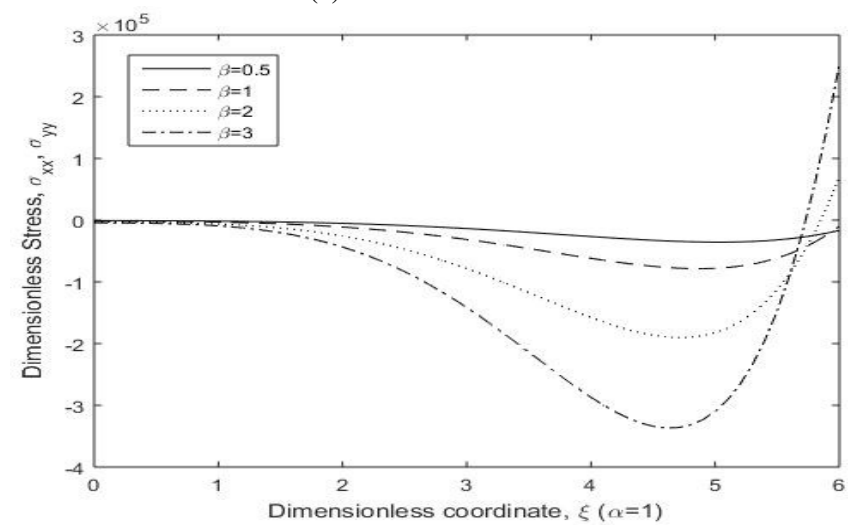

(d) $\alpha=1$

Figure 7: Dimensionless Stress distribution with space variable $\xi$ for different values of thermal conductivity parameter $\beta$ and fractional order $\alpha$.

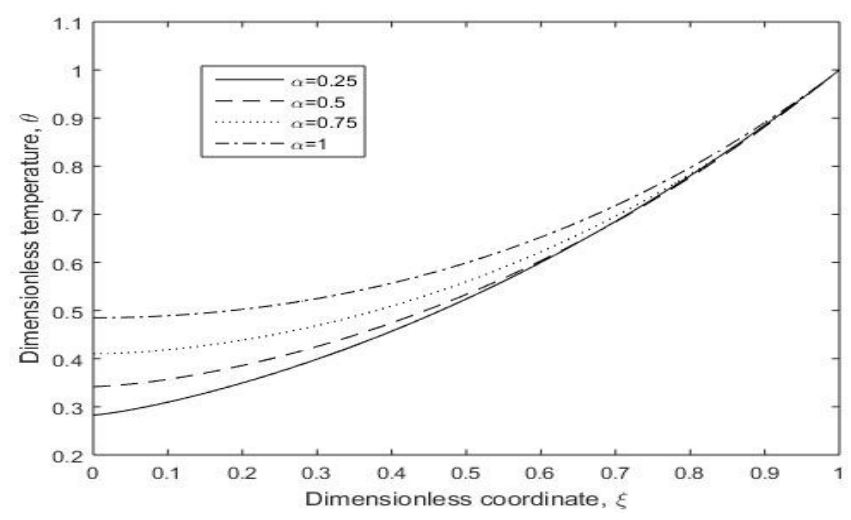

(a)

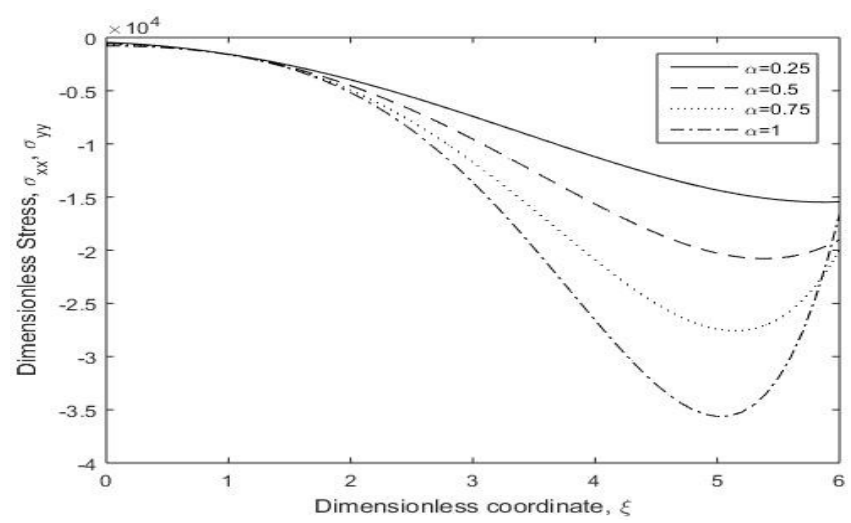

(b)

Figure 8: Effect of various fraction order parameters $\alpha$ on (a) dimensionless temperature distribution (b) dimensionless Stress distribution with space variable $\xi$.

Figure $2 \mathrm{a}-2 \mathrm{~d}$, shows that the temperature distribution of rectangular fin with respect to space variable $\xi$ for different values of thermo-geometric parameter $\phi=1,2,3,4$ and with various fractional orders $\alpha=0.25,0.5,0.75,1$ by keeping convection parameter $\beta$ fixed at 1 . From the graphs it is found that the fractional order $\alpha$ represents the point of convergence for the given range of intervals between 0 to 1 . Figure $3 \mathrm{a}-3 \mathrm{~d}$ represents the temperature distribution with $\xi$ for various thermal conductivity parameters $\beta=0.5,1,2,3$ keeping $\phi$ value constant at 1 . It is noted that for higher convection parameters the gradual drop of temperature is experienced and the fin suddenly reaches the surrounding temperature. It is also observed that as the value of fractional order $\alpha$ increases the temperature of fins goes on increasing. In Figure 4 the fin efficiency with thermo-geometric fin parameter $\phi$ for different values of fractional order $\alpha=0.25,0.5,0.75,1$ has been studied by keeping $\beta=1$, then the retarded drop in fin efficiency is observed with the rise of fractional order parameter $\alpha$. The similar observations made for fin effectiveness with thermogeometric fin parameter $\phi$ for different parameter values of $\alpha$ by taking constant value $\beta=1, \psi=1$ in Figure 5a. Whereas in Figure $5 \mathrm{~b}$ we plotted fin effectiveness with thermo-geometric fin parameters $\beta=1, \alpha=1$ and varying different values of fin length/fin thickness ratio $\psi=1,2,3,4$. Figure 6a-6d shows that as the thermogeometric fin parameter increases the stress decreases while from Figure $7 \mathrm{a}-7 \mathrm{~d}$ it is observed that as the thermal conductivity and fractional parameters increase the stresses reduced. Figure $8 \mathrm{a}-8 \mathrm{~b}$ shows the effect of fractional order $\alpha$ on temperature and stresses. Here remaining parameters are constant $\phi=1$ and $\beta=0.5$. We have observed that as the $\alpha$ rises the temperature increases and stresses decreases.

\section{Concluding Remarks}

In this work, the fractional order energy balance equation has been introduced to study the thermal stresses 
in a rectangular profile fin with temperature dependent thermal conductivity. HPM is used to obtain the solution in thermal domain. This method is based on perturbation iterative technique, a useful method to obtain the solution of non-linear fractional equations. This method does not provide an exact analytic solution as it contains infinite power series terms but the obtained results are enough to come to a conclusion about the efficiency of the method. The motivation behind the consideration of the fractional theory is that it predicts retarded response to physical stimuli. It is concluded that for different values of the fractional order parameter $\alpha$ the propagation of the wave changes. The fractional parameter seems to be directly proportional to the temperature distribution of the material (see in Table 1). The phenomena reveal that the selection of the order of fractional derivatives remarkably influences the outcomes. Mathematical modeling is provided for the nondimensional thermal stress in fins that leads to a new approach in investigation of the extended surface base problems. The results of this investigation may be very helpful for engineers dealing with the thermal stress problems of linear or nonlinear nature.

\section{Acknowledgment}

Author would like to thank Prof. Dr. Ganesh D. Kedar of the Department of Mathematics, Rashtrasant Tukadoji Maharaj Nagpur University, Nagpur (India) for their assistance that greatly improved this manuscript.

\section{References}

[1] A. Aziz and S. M. Enamul Huq. "Perturbation solution for convecting fin with variable thermal conductivity", pp. 300-301,1975.

[2] S. Ma, A. Behbahani, and Y. Tsuei, "Two-dimensional rectangular fin with variable heat transfer coefficient," International Journal of Heat and Mass Transfer, vol. 34, no. 1, pp. 79-85, 1991.

[3] S. Baskaya, M. Sivrioglu, and M. Ozek, "Parametric study of natural convection heat transfer from horizontal rectangular fin arrays," International Journal of Thermal Sciences, vol. 39, no. 8, pp. 797-805, 2000.

[4] E. M. Mokheimer, "Performance of annular fins with different profiles subject to variable heat transfer coefficient," International Journal of Heat and Mass Transfer, vol. 45, no. 17, pp. 3631-3642, 2002.

[5] S. A. El-Sayed, S. M. Mohamed, A. M. Abdellatif, and E. A. Abdelhamid, "Investigation of turbulent heat transfer and fluid flow in longitudinal rectangular fin arrays of different geometries and shrouded fin array," Experimental Thermal and Fluid Science, vol. 26, no. 8, pp. 879-900, 2002.

[6] C.-H. Chiu and C.-K. Chen, "Application of adomian's decomposition procedure to the analysis of convective radiative fins," J. Heat Transfer, vol. 125, no. 2, pp. 312-316, 2003.

[7] C. Arslanturk, "A decomposition method for fin efficiency of convective straight fins with temperature dependent thermal conductivity," International
Communications in Heat and Mass Transfer, vol. 32, no. 6, pp. 831-841, 2005.

[8] S. B. Coskun and M. T. Atay, "Analysis of convective straight and radial fins with temperature dependent thermal conductivity using variational iteration method with comparison with respect to finite element analysis," Mathematical Problems in Engineering, vol. 2007, 2007.

[9] K. Pitchandi and E. Natarajan, "Entropy generation in pin fins of circular and elliptical cross sections in forced convection with air," International Journal of Thermodynamics, vol. 11, no. 4, pp. 161-171, 2008.

[10] G. Domairry and M. Fazeli, "Homotopy analysis method to determine the fin efficiency of convective straight fins with temperature dependent thermal conductivity," Communications in Nonlinear Science and Numerical Simulation, vol. 14, no. 2, pp. 489 499, 2009.

[11] E. Cuce and P. M. Cuce, "Homotopy perturbation method for temperature distribution, fin efficiency and fin effectiveness of convective straight fins with temperature dependent thermal conductivity," Proceedings of the Institution of Mechanical Engineers, Part C: Journal of Mechanical Engineering Science, vol. 227, no. 8, pp. 1754-1760, 2013.

[12] A. Sciacovelli, E. Guelpa, and V. Verda, "Second law optimization of a pcm based latent heat thermal energy storage system with tree shaped fins," International Journal of Thermodynamics, vol. 17, no. 3, pp. 145-154, 2014.

[13] G.J. Huang, S.C. Wong, and C.P. Lin, "Enhancement of natural convection heat transfer from horizontal rectangular fin arrays with perforations in fin base," International Journal of Thermal Sciences, vol. 84, pp. 164-174, 2014.

[14] A. Mallick, R. Ranjan, and P. K. Sarkar, "Effect of heat transfer on thermal stresses in an annu-lar hyperbolic fin: an approximate analytical solu-tion," Journal of Theoretical and Applied Mechan-ics, vol. $54,2016$.

[15] I. Podlubny, "Fractional differential equations, mathematics in science and engineering," 1999.

[16] M. Caputo, "Linear models of dissipation whose $q$ is almost frequency independentii," Geophysical Journal International, vol. 13, no. 5, pp. 529-539, 1967.

[17] M. Caputo, Elasticita e dissipazione. Zanichelli, 1969.

[18] M. Caputo and F. Mainardi, "Linear models of dissipation in anelastic solids," La Rivista del Nuovo Cimento (1971-1977), vol. 1, no. 2, pp. 161-198, 1971. 
[19] H. H. Sherief and A. Abd El-Latief, "Application of fractional order theory of thermoelasticity to a $1 \mathrm{~d}$ problem for a half-space," ZAMM-Journal of Applied Mathematics and Mechanics/Zeitschrift f"ur Angewandte Mathematik und Mechanik, vol. 94, no. 6, pp. 509-515, 2014.

[20] Y. Povstenko, Fractional thermoelasticity, vol. 219. Springer, 2015.

[21] X.-Y. Zhang, Z.-T. Chen, and X.-F. Li, "Thermal shock fracture of an elastic half space with a subsurface penny shaped crack via fractional thermoelasticity," Acta Mechanica, vol. 229, no. 12, pp. 4875-4893, 2018.

[22] Y. Povstenko and T. Kyrylych, "Fractional thermoelasticity problem for an infinite solid with a penny shaped crack under prescribed heat flux across its surfaces," Philosophical Transactions of the Royal Society A, vol. 378, no. 2172, p. 20190289, 2020.

[23] V. Srinivas, V. Manthena, J. Bikram, and G. Kedar, "Fractional order heat conduction and thermoelastic response of a thermally sensitive rectangular parallelopiped," International Journal of Thermodynamics, vol. 24, no. 1, pp. 62-73, 2021.

[24] T. Patel and R. Meher, "A study on temperature distribution, efficiency and effectiveness of longitudinal porous fins by using adomian decomposition sumudu transform method," Procedia Engineering, vol. 127, pp. 751-758, 2015.

[25] A. Patra and S. S. Ray, "Analysis for fin efficiency with temperature dependent thermal conductivity of fractional order energy balance equation using hpst method," Alexandria Engineering Journal, vol. 55, no. 1, pp. 77-85, 2016.

[26] D. Kumar, J. Singh, and D. Baleanu, "A new fractional model for convective straight fins with temperature-dependent thermal conductivity," 2018.

[27] Y. Yener and S. Kakac, "Heat conduction", taylor and francis," 2008.
[28] F.J. Liu, Z.-B. Li, S. Zhang, and H.-Y. Liu, "He's fractional derivative for heat conduction in a fractal medium arising in silkworm cocoon hierarchy," Thermal Science, vol. 19, no. 4, pp. 1155-1159, 2015.

[29] K. S. Miller and B. Ross, "An introduction to the fractional calculus and fractional differential equations",Wiley, 1993.

[30] J.H. He, "Homotopy perturbation technique," Computer methods in applied mechanics and engineering, vol. 178, no. 3-4, pp. 257-262, 1999.

[31] J. H. He, "A coupling method of a homotopy technique and a perturbation technique for non-linear problems," International journal of non-linear mechanics, vol. 35, no. 1, pp. 37-43, 2000.

[32] J.H. He, "Homotopy perturbation method for solving boundary value problems," Physics letters A, vol. 350, no. 1-2, pp. 87-88, 2006.

[33] N. Noda, R. Hetnarski, and Y. Tanigawa, "Thermal stresses, taylor \& francis," New York, vol. 260, 2003.

[34] W. Nowacki, Thermoelasticity. Elsevier, 2013. 